\title{
Os Hospitais e a Combinação Público-Privado no Sistema de Saúde Português
}

\author{
Hospitals and the Public-Private Combination in the \\ Portuguese Health System
}

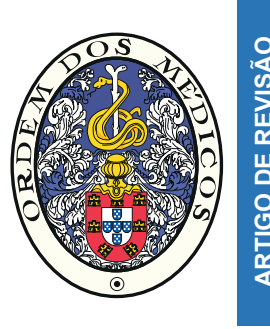

\author{
Adalberto Campos FERNANDES ${ }^{1}$, Alexandre Morais NUNES $\square^{2}$ \\ Acta Med Port 2016 Mar;29(3):217-223 - http://dx.doi.org/10.20344/amp.6712
}

RESUMO

O sistema de saúde português tem sido caraterizado pela existência de uma permanente relação entre o setor público e o setor privado tanto na prestação como no financiamento dos cuidados de saúde. Nas últimas décadas, ao nível da prestação de cuidados o setor privado aumentou significativamente a sua capacidade de resposta ampliando o seu nível de envolvimento na relação com o setor público. Esta relação decorre do enquadramento jurídico definido na Lei de Bases da Saúde desenvolvendo-se sob a forma de acordos, convenções e contratos bem como, mais recentemente, através do modelo de parcerias-público privadas. No que se refere ao caso específico da rede hospitalar esta nova dinâmica de relação contribuiu, nas últimas duas décadas, para acentuar a natureza mista do sistema através de um evidente reforço da componente privada na rede hospitalar particularmente através do investimento em unidades de âmbito generalista.

Palavras-chave: Hospitais; Serviço Nacional de Saúde; Parcerias Público-Privadas;Portugal; Sector Privado; Sector Público.

\section{ABSTRACT}

The Portuguese health system has been characterized by the existence of a constant relationship between public and private sector, both in providing and financing health care. In recent decades, the private sector increased their responsiveness of care, extending the engagement in the relationship with the public sector. This relationship stems from the legal framework set out in the law, developing agreements, conventions and more recently through the model of public-private partnerships. In hospital network, this new dynamic relationship contributed, in the last two decades, to accentuate the mixed characteristics of the system, through a clear strengthening of the private component in the hospital network, particularly by investing in differentiated units.

Keywords: Hospitals; National Health Service Portugal; Private Sector; Public Health; Public-Private Sector Partnerships.

\section{INTRODUÇÃO}

O sistema de saúde, em Portugal carateriza-se por uma cobertura universal (em termos da população servida) e geral (em termos das especialidades oferecidas), de matriz predominante pública, integrando financiamento e prestação repartida por três segmentos fundamentais - cuidados hospitalares, cuidados de saúde primários e cuidados continuados integrados. Apesar da presença dominante, ao nível do financiamento e da prestação, mantida desde a criação do Serviço Nacional de Saúde (SNS), a participação do setor privado, nos últimos anos, tem vindo a ser crescente nos diferentes níveis do financiamento e da prestação de cuidados. ${ }^{1}$

No âmbito da combinação público-privada têm sido múltiplos os mecanismos de cooperação entre os dois setores. No essencial podem ser distinguidos dois tipos de relação: um de natureza complementar - em que os cidadãos recorrem a prestadores privados mediante acordo estabelecido com os serviços públicos - e outro de substituição, em que há lugar à utilização dos serviços privados por iniciativa própria. Em qualquer das situações o objetivo é garantir o acesso ao sistema de saúde².

Este recurso do SNS aos regimes de complementaridade ou de substituição, no que se refere à prestação de cuidados de saúde, tem funcionado como um incentivo ao setor privado no que diz respeito ao seu modelo de desenvolvimento estratégico. Esta interdependência tem contribuído, igualmente, para que os prestadores privados desenvolvam modelos incentivadores de maior eficiência e de maior equidade quando encarados como substitutos da função de prestação do Serviço Nacional de Saúde. ${ }^{3}$

Ao longo da evolução do SNS, a questão da complementaridade e da concorrência, entre prestadores públicos e privados tem sido objeto de um vasto acervo de investigação, avaliação e análise. Num estudo realizado em Portugal, por Oliveira ${ }^{4}$ denominado "Evolução da oferta", foi reconhecida a existência de uma complementaridade produtiva, na medida em que o SNS centrava a sua ação predominante nos cuidados hospitalares agudos, nos cuidados de saúde primários e nos cuidados materno-infantis, ao passo que a iniciativa privada apresentava uma grande incidência nas consultas de especialidade, de medicina dentária e estomatologia e nos meios de diagnóstico e de terapêutica. ${ }^{4}$

Neste artigo, abordam-se as diferentes formas de combinação público-privada, tendo em conta o atual enquadramento legal, analisando-se a relação entre o desenvolvimento da rede hospitalar pública e privada e a repartição das unidades pelas regiões administrativas, no período compreendido entre os anos de 2002 e 2012.

\footnotetext{
1. Departamento de Gestão em Saúde e de Políticas de Saúde. Escola Nacional de Saúde Pública. Universidade NOVA de Lisboa. Lisboa. Portugal.

2. Departamento de Políticas de Saúde. Instituto Superior de Ciências Sociais e Políticas. Universidade de Lisboa. Lisboa. Portugal.

$\triangle$ Autor correspondente: Alexandre Morais Nunes. alexandre_morais_nunes@hotmail.com

Recebido: 06 de junho de 2015 - Aceite: 28 de dezembro de 2015 | Copyright $\odot$ Ordem dos Médicos 2016
} 


\section{As formas legais de relação público-privado}

De acordo com o princípio constitucional inscrito no ar-

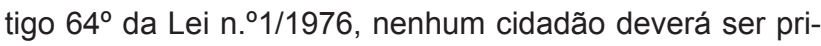
vado do direito à proteção na saúde e à prestação de cuidados no momento em que deles precise, independentemente da sua condição económica e social. Este princípio constitucional carateriza a ação dos prestadores privados como sendo de natureza complementar ao SNS referindo que os objetivos desta articulação passam por assegurar, nas instituições de saúde públicas e privadas, adequados padrões de eficiência e de qualidade. ${ }^{5}$

Apesar dos diferentes acordos estabelecidos na década de 80 , entre o setor público e o setor privado, foi a partir de 1993, com a publicação da Lei de Bases da Saúde (LBS), que foram legisladas as formas de relação entre a prestação pública e privada nos cuidados de saúde. Estas relações passaram a efetivar-se através da celebração de acordos, da contratação de serviços e da realização de convenções.

Em simultâneo, o Estado acentuou a posição de regulador da atividade, numa perspetiva de garantir o acesso aos serviços a toda a população tendo presente a salvaguarda da qualidade dos cuidados. ${ }^{6}$ Na prática o Estado assumiu uma posição repartida entre a prestação direta e um maior foco na regulação, salvaguardando o cumprimento das bases constitucionais através de uma prestação de cuidados assegurada por entidades públicas e privadas promotora da equidade, da universalidade e da qualidade a todos os cidadãos. .,7,8 $^{2}$

O reforço das funções reguladoras, por parte do Estado, não significou, no entanto, a diminuição das suas responsabilidades e participação na prestação de cuidados de saúde. As atividades de regulação, acompanhamento, fiscalização e controlo não diminuíram a função de principal prestador do serviço público. ${ }^{9}$

\section{O desenvolvimento do setor privado na prestação de cuidados de saúde}

O SNS domina a prestação de cuidados de saúde em Portugal, desde o momento da sua criação em 1979, embora se verifique um quadro de progressiva complementaridade com os prestadores privados..$^{10,11} \mathrm{~A}$ prestação pública predomina nos cuidados de saúde primários e hospitalares enquanto a prestação privada tem vindo a adquirir uma importância crescente em algumas áreas específicas (nefrologia - diálise renal, ginecologia, saúde oral, ortopedia e pediatria), ao nível dos meios complementares de diagnóstico e de terapêutica (imagiologia, fisioterapia e patologia clínica) e no setor do medicamento (farmácias comunitárias)..$^{12,13}$

Campos e Simões ${ }^{2}$ associam os dados referentes ao crescimento destas áreas aos avanços tecnológicos ocorridos nos últimos cinquenta anos e ao seu uso massificado em regime de ambulatório. Os autores destacam que até aos anos 90 , a maioria das unidades privadas eram propriedade ou estavam dirigidas por profissionais do SNS gerando uma competição adversa com o setor público, ao nível do acesso, através da indução da atividade no setor privado.

O crescimento da oferta do setor privado resultou de um aumento da procura gerada, em grande parte, por uma maior limitação da oferta pública. Este facto contribuiu para o reforço do investimento em novas infraestruturas com o consequente acréscimo na participação do setor privado ao nível da prestação dos serviços. A cooperação entre os setores tornou-se necessária para o SNS tendo em vista o cumprimento das obrigações de cobertura geral e acesso universal a toda a população. Contribuiu, igualmente, para corrigir as falhas existentes permitindo dar uma resposta mais adequada, atempada e de qualidade aos seus utentes, através das instituições de direito privado. ${ }^{14,15}$

$\mathrm{Na}$ base deste acréscimo de procura pela oferta privada encontram-se diferentes tipos de falhas do setor público tais como: défice de cobertura; tempos de espera prolongados para a marcação e a realização de exames; a existência de um grande número de cidadãos sem médico de família; ineficiências do SNS na integração vertical entre os cuidados de saúde geradoras de listas de espera (marcação de cirurgias e de primeiras consultas hospitalares de especialidade)..$^{15}$

O dinamismo do setor privado resultou, igualmente, das condições de autonomia de gestão face à prestação pública, da capacidade de investimento na inovação tecnológica, nomeadamente, em equipamentos de diagnóstico e terapêutica. ${ }^{16,17}$ Desta forma, as instituições privadas tornaram-se mais competitivas na captação de novos utentes. ${ }^{18}$ Em certa medida, o Estado através do exercício das suas próprias políticas acabou por induzir o recurso ao setor privado através de diferentes meios:

- Implementação de programas especiais de recuperação de listas de espera (com recursos à cooperação privada);

- Implementação de um sistema de incentivos fiscais deduções para gastos em saúde;

- Incentivo à instalação e desenvolvimento de instituições privadas;

- Incentivo à contratualização do setor privado pelo Estado;

- Incentivo à mobilidade do pessoal do SNS para o setor privado decorrente da possibilidade dos profissionais de saúde aumentarem o seu rendimento através desta atividade. ${ }^{4}$

\section{Relação de oportunidade}

Os setores público e privado na prestação de cuidados de saúde, na sua orgânica e na estrutura do seu funcionamento, não são independentes nem estão isolados. Existe uma mútua influência no desenvolvimento destes setores no que concerne a aspetos como a procura de eficiência, a otimização de meios e de recursos e o fomento da qualidade. ${ }^{2}$

No que se refere ao setor público a estratégia adotada, nos últimos 30 anos, teve como intuito fundamental a criação de um modelo de gestão baseado em práticas 
empresariais que ganhou maior relevo a partir do ano 2002 com a publicação da Lei n. ${ }^{\circ} 27 / 2002$ de 8 de novembro. O setor privado reforçou o seu crescimento beneficiando das insuficiências no desempenho do setor público procurando nas suas falhas, limitações ou resultados menos positivos, uma oportunidade de crescimento. Este processo desenvolveu-se numa relação de oportunidade sustentada numa prática competitiva que acabou por contribuir para a inovação do sistema de saúde estimulando, desse modo, uma concorrência positiva entre as unidades públicas e privadas. $^{2}$

A competição entre os prestadores de cuidados de saúde, públicos e privados, contemplada na Lei de Bases da Saúde, prevê o desenvolvimento do setor privado e social, em concorrência com o setor público, apelando às vantagens potenciais que poderão advir desta relação. No entanto, é importante ter presente que os critérios de controlo da qualidade, o nível de requisitos nos padrões de acesso e dos resultados aplicados à regulação no setor da saúde, deverão ser os mesmos, tanto para os prestadores públicos como para os privados. ${ }^{19}$

\section{Áreas de cooperação}

São múltiplas as áreas de cooperação entre os dois setores nas quais o Estado recorre ao setor privado a fim de assegurar determinada atividade assistencial ou reali- zar determinados programas de saúde para os quais não tem capacidade de execução. Como exemplos deste tipo de relações, entre os dois setores, podem ser referidos o sistema integrado de gestão das listas de espera cirúrgicas - denominado SIGIC (Sistema Integrado de Gestão de Inscritos para Cirurgia); o programa cheque dentista para grávidas, crianças, idosos; o tratamento a doentes portadores de infeção por VIH/sida; a Rede Nacional de Cuidados Continuados Integrados; bem como as Parcerias Público-Privadas (PPP).

Para além da cooperação, existe uma relação aparentemente geradora de benefícios para ambos os setores. Pelo lado do Estado, este consegue, com o recurso ao setor privado e social cumprir a função de garante de proteção na saúde à população enquanto o setor privado consegue captar novos utentes apostando na complementaridade com o sistema público, o que favorece o seu desenvolvimento ao nível da oferta de cuidados de saúde no contexto do sistema de saúde.

\section{O desenvolvimento da rede hospitalar}

A rede hospitalar em Portugal sofreu um conjunto de importantes alterações nos últimos 40 anos, não só em termos de vínculo institucional como também no seu estatuto jurídico. Portugal integra um grupo de países onde coexistem hospitais públicos com um extenso setor privado. ${ }^{20}$

Tabela 1 - Distribuição dos hospitais portugueses, segundo a natureza institucional, entre 1970 e 2012

\begin{tabular}{|c|c|c|c|c|c|}
\hline Ano & Total & Hospital Oficial (n) & Hospital Oficial (\%) & Hospital Privado (n) & Hospital Privado (\%) \\
\hline 1970 & 615 & 171 & 27,8 & 444 & 72,2 \\
\hline 1975 & 532 & 185 & 34,8 & 347 & 65,2 \\
\hline 1980 & 491 & 394 & 80,2 & 97 & 19,8 \\
\hline 1985 & 232 & 139 & 59,9 & 93 & 40,1 \\
\hline 1990 & 240 & 145 & 60,4 & 95 & 39,6 \\
\hline 1995 & 200 & 119 & 59,5 & 81 & 40,5 \\
\hline 2000 & 219 & 125 & 57,1 & 94 & 42,9 \\
\hline 2001 & 217 & 122 & 56,2 & 95 & 43,8 \\
\hline 2002 & 213 & 119 & 55,9 & 94 & 44,1 \\
\hline 2003 & 204 & 114 & 55,9 & 90 & 44,1 \\
\hline 2004 & 209 & 117 & 56,0 & 92 & 44,0 \\
\hline 2005 & 204 & 112 & 54,9 & 92 & 45,1 \\
\hline 2006 & 200 & 107 & 53,5 & 93 & 46,5 \\
\hline 2007 & 198 & 99 & 50,0 & 99 & 50,0 \\
\hline 2008 & 189 & 92 & 48,7 & 97 & 51,3 \\
\hline 2009 & 186 & 86 & 46,2 & 100 & 53,8 \\
\hline 2010 & 229 & 127 & 55,5 & 102 & 44,5 \\
\hline 2011 & 226 & 123 & 54,4 & 103 & 45,6 \\
\hline 2012 & 214 & 110 & 51,4 & 104 & 48,6 \\
\hline
\end{tabular}

Fonte: INE (2014) ${ }^{21}$; PORDATA (2013) ${ }^{23}$ 


\section{Evolução do parque hospitalar português}

Em 1970 existiam 615 hospitais em Portugal, que na sua maioria se encontravam associados às diferentes Misericórdias. ${ }^{21}$ Em 1979, com a criação do SNS a sua propriedade foi transferida para o Estado. Nos anos 90 estas unidades foram integradas nos hospitais distritais enquanto outras unidades de menor dimensão - os hospitais conceIhios foram transformados em centros de saúde. Com estas alterações houve uma diminuição sensível do número total de hospitais em Portugal..$^{15,22}$

O ano de 2002 foi caraterizado pela introdução de alterações importantes na organização e na gestão hospitalar tendo em vista a adoção de práticas de gestão empresarial. Neste sentido, foram transformadas em sociedades anónimas cerca de 31 unidades hospitalares que integravam a rede pública do SNS. Em 2005, foi realizada uma nova alteração do estatuto jurídico dos hospitais alterando o estatuto de sociedade anónima para Entidades Públicas Empresariais. ${ }^{24-26} \mathrm{~A}$ partir de 2005 , teve início um processo de fusão e de concentração de unidades hospitalares com o objetivo de promover uma melhor gestão dos recursos técnicos e financeiros sem pôr em causa a qualidade ou o acesso aos serviços. ${ }^{24,27}$

A Tabela 1 apresenta a evolução do número de hospitais no sistema de saúde, em termos totais, ou discriminados pela natureza institucional (oficiais ou privados), desde o ano de 1970 até 2012.

A Figura 1 mostra a evolução da rede hospitalar do sistema de saúde português, entre os anos 1970 e 2012.

No período de tempo compreendido entre 2002 e 2012, o número total de unidades hospitalares permaneceu praticamente inalterado. Quando se faz uma análise em termos de estatuto observa-se uma redução das unidades públicas (em nove unidades) e um aumento das unidades privadas (em dez unidades). Em termos percentuais, no ano de 2012 os hospitais privados representavam $48,6 \%$ da oferta hospitalar evidenciando um crescimento de $4,5 \%$ em 10 anos.

\section{Distribuição geográfica das unidades hospitalares}

A evolução do parque hospitalar sofreu variações, ao longo dos anos, como se observou no ponto anterior. Tendo presente o objetivo de avaliar a importância da rede hospitalar em termos do setor público ou do setor privado nas várias regiões do país analisámos de seguida a evolução dos hospitais em termos da distribuição geográfica destas unidades. A Tabela 2 reflete a distribuição dos hospitais portugueses pelas cinco regiões administrativas.

Com base nos dados apurados observam-se diferentes proporções na distribuição dos hospitais oficiais (públicos) e dos hospitais privados nas diferentes regiões do país, entre os anos de 1999 e 2012. Desta forma verificámos os seguintes pontos:

- A região Norte do País regista uma variação positiva da oferta privada, atingindo o maior valor em termos percentuais no ano de 2003 (com um diferencial de 10,2\% relativamente ao público, no mesmo ano);

- A região Centro regista um predomínio dos hospitais oficiais, em 2003 , com uma proporção de $11,5 \%$ superior aos hospitais privados;

- Ao nível de Lisboa e Vale do Tejo registam-se duas tendências de comportamento opostas. No início do período considerado, entre os anos de 1999 e 2003, existe um aumento da proporção de hospitais privados, com um diferencial de $6 \%$ e $4,4 \%$, respetivamente. Posteriormente, nos anos de 2006, 2009 e 2012 seguiu-se um período de predomínio dos hospitais oficiais (com um maior diferencial em 2006, com 4,7\%);

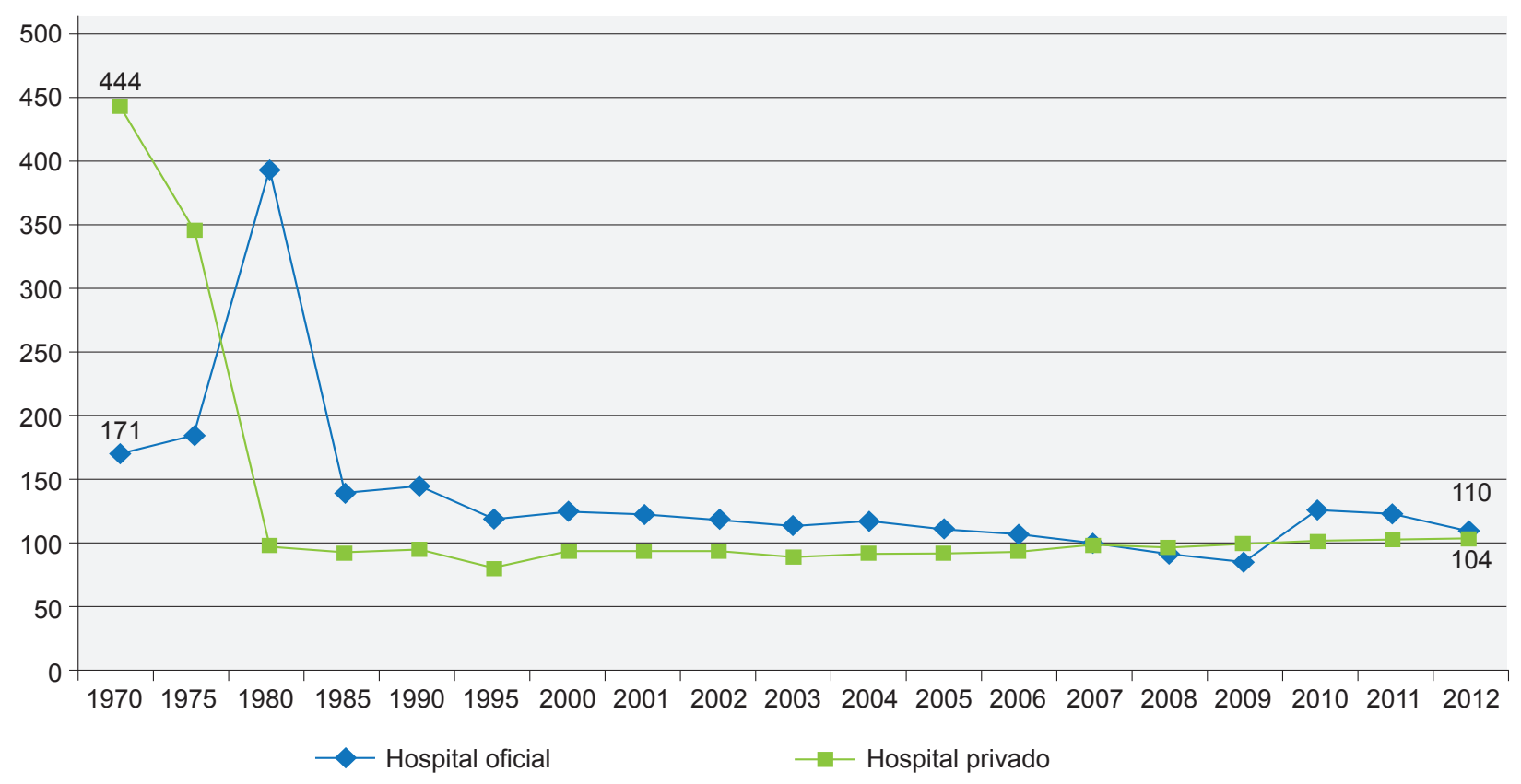

Figura 1 - Evolução da rede hospitalar em Portugal, entre 1970 e 2012 Fonte: INE (2014) ${ }^{21}$ 
Tabela 2 - Hospitais do sistema de saúde português, por localização geográfica (regiões de Portugal Continental) e natureza institucional

\begin{tabular}{|c|c|c|c|c|c|c|c|c|c|c|c|c|c|c|c|}
\hline & & 1999 & & & 2003 & & & 2006 & & & 2009 & & & 2012 & \\
\hline & Total & $\mathrm{HO}$ & HP & Total & $\mathrm{HO}$ & $\mathrm{HP}$ & Total & $\mathrm{HO}$ & $\mathrm{HP}$ & Total & $\mathrm{HO}$ & HP & Total & $\mathrm{HO}$ & HP \\
\hline Continente (n) & 190 & 98 & 92 & 171 & 82 & 89 & 185 & 103 & 82 & 189 & 110 & 79 & 205 & 121 & 84 \\
\hline Norte (\% total) & 36,3 & 34,7 & 38,0 & 33,3 & 28,0 & 38,2 & 35,1 & 32,0 & 39,0 & 32,3 & 30,9 & 34,2 & 31,7 & 31,4 & 32,1 \\
\hline Norte (n) & 79 & 34 & 35 & 57 & 23 & 34 & 65 & 33 & 32 & 61 & 34 & 27 & 65 & 38 & 27 \\
\hline Norte (\%) & 100 & 49,3 & 50,7 & 100 & 40,4 & 59,6 & 100 & 50,8 & 49,2 & 100 & 55,7 & 44,3 & 100 & 58,5 & 41,5 \\
\hline Centro (\% total) & 25,3 & 29,6 & 20,7 & 25,7 & 31,7 & 20,2 & 24,9 & 30,1 & 18,3 & 25,9 & 28,2 & 22,8 & 20,5 & 22,3 & 17,9 \\
\hline Centro (n) & 48 & 29 & 19 & 44 & 26 & 18 & 46 & 31 & 15 & 49 & 31 & 18 & 42 & 27 & 15 \\
\hline Centro (\%) & 100 & 60,4 & 39,6 & 100 & 59,1 & 40,9 & 100 & 67,4 & 32,6 & 100 & 63,3 & 36,7 & 100 & 64,3 & 35,7 \\
\hline Lisboa (\% total) & 28,4 & 25,5 & 31,5 & 31,6 & 29,3 & 33,7 & 30,3 & 28,2 & 32,9 & 31,7 & 30,0 & 34,2 & 39,0 & 37,2 & 41,7 \\
\hline Lisboa (n) & 54 & 25 & 29 & 54 & 24 & 30 & 56 & 29 & 27 & 60 & 33 & 27 & 80 & 45 & 35 \\
\hline Lisboa (\%) & 100 & 46,3 & 53,7 & 100 & 44,4 & 55,6 & 100 & 51,8 & 48,2 & 100 & 55,0 & 45,0 & 100 & 56,3 & 43,8 \\
\hline Alentejo ( $\%$ total) & 5,3 & 6,1 & 4,3 & 5,3 & 7,3 & 3,4 & 5,4 & 6,8 & 3,7 & 5,8 & 7,3 & 3,8 & 4,9 & 5,8 & 3,6 \\
\hline Alentejo (n) & 10 & 6 & 4 & 9 & 6 & 3 & 10 & 7 & 3 & 11 & 8 & 3 & 10 & 7 & 3 \\
\hline Alentejo (\%) & 100 & 60,0 & 40,0 & 100 & 66,7 & 33,3 & 100 & 70,0 & 30,0 & 100 & 72,7 & 37,5 & 100 & 70,0 & 30,0 \\
\hline Algarve (\% total) & 4,7 & 4,1 & 5,4 & 4,1 & 3,7 & 4,5 & 4,3 & 2,9 & 6,1 & 4,2 & 3,6 & 5,1 & 3,9 & 3,3 & 4,8 \\
\hline Algarve $(n)$ & 9 & 4 & 5 & 7 & 3 & 4 & 8 & 3 & 5 & 8 & 4 & 4 & 8 & 4 & 4 \\
\hline Algarve (\%) & 100 & 44,4 & 55,6 & 100 & 42,9 & 57,1 & 100 & 37,5 & 62,5 & 100 & 50,0 & 50,0 & 100 & 50,0 & 50,0 \\
\hline
\end{tabular}

HP - hospitais privados: $\mathrm{HO}$ - hospitais oficiais

Fonte: INE (2014) $)^{21}$; PORDATA(2013) 23

- A região do Alentejo, ao longo de todo o período considerado, registou um predomínio dos hospitais oficiais;

- A região do Algarve verificou uma situação oposta ao longo do período em análise com uma predominância do número de unidades privadas.

Quando analisada a relação entre os hospitais públicos e privados é importante estabelecer a sua proporção em termos dos totais no sistema de saúde. Considerando todos os hospitais do sistema de saúde, verifica-se que a região Norte do país apresentou um maior número de hospitais até ao ano de 2009, a partir do qual se começou a registar uma diminuição. Por sua vez, a região de Lisboa e Vale do Tejo, a partir do ano 2009 apresentou um aumento do número de unidades hospitalares. Na região Centro, os hospitais apresentaram uma proporção praticamente constante até 2009 , período a partir do qual registaram uma diminuição.

Avaliando a rede hospitalar uma forma global e dado que foram registadas algumas transformações relativas à distribuição pelas Administrações Regionais de Saúde é interessante confrontar estas conclusões em termos da natureza institucional das instituições.

Ao nível do setor privado, registaram-se como evoluções:

- Na região Norte: um crescimento até ao ano 2006 e a partir daí uma descida considerável de 6,9\%;

- Na região de Lisboa e Vale do Tejo: a partir de 2006 registou-se um crescimento de $8,8 \%$ ocorrendo um ponto de viragem em 2009, momento a partir do qual esta região superou o Norte do país;

- Na zona Centro destaca-se um período de crescimento entre os anos 2006 e 2009 de $4,5 \%$, ao qual se seguiu uma descida de 4,9\%;

- Na região do Alentejo: o investimento em unidades privadas apresentou um pico máximo de 6,1\% em 2006 e uma ligeira quebra até 2012;

- No Algarve: a predominância privada é praticamente constante, sem grandes oscilações.

Ao nível do setor público registaram-se as seguintes evoluções:

- Na região Norte: uma redução do número de unidades de 1999 a 2003 e posteriormente uma subida até 2006 , tendo a partir desta data apresentado pequenas oscilações. No período considerado estas unidades, em termos da representação no total nacional viram a sua percentagem reduzida em 3,3\%;

- Na região de Lisboa e Vale do Tejo: a proporção de unidades hospitalares oficiais subiu $11,7 \%$;

$\mathrm{Na}$ zona Centro: destaca-se um ligeiro crescimento na representação destas unidades a nível nacional de 1999 a 2003 , de $2,1 \%$, ao qual se seguiu uma tendência de redução de 9,4\%;

- Na região do Alentejo: a proporção dos hospitais públicos reduziu-se apenas em $0,3 \%$;

- No Algarve: a rede pública decresceu cerca de 0,8\%.

- No ano de 2009 na região Norte verifica-se uma 
Tabela 3 - Número de camas disponíveis nos hospitais, por localização geográfica (regiões de Portugal Continental) e natureza institucional (por 100000 habitantes)

\begin{tabular}{|c|c|c|c|c|c|c|c|c|}
\hline & \multicolumn{2}{|c|}{2004} & \multicolumn{2}{|c|}{2005} & \multicolumn{2}{|c|}{2006} & \multicolumn{2}{|c|}{2007} \\
\hline & $\mathrm{HO}$ & $\mathrm{HP}$ & $\mathrm{HO}$ & $\mathrm{HP}$ & $\mathrm{HO}$ & $\mathrm{HP}$ & $\mathrm{HO}$ & $\mathrm{HP}$ \\
\hline Norte & 230,8 & 71,3 & 229,9 & 71,8 & 216,1 & 84,2 & 212,7 & 79,0 \\
\hline Centro & 314,7 & 55,1 & 310,9 & 54,6 & 302,4 & 39,5 & 302,8 & 39,9 \\
\hline Lisboa & 329,1 & 108,9 & 323,0 & 107,0 & 313,3 & 106,4 & 299,7 & 115,7 \\
\hline Alentejo & 192,3 & 31,8 & 197,0 & 31,9 & 196,1 & 28,2 & 190,6 & 30,8 \\
\hline Algarve & 191,1 & 18,7 & 184,1 & 17,6 & 183,7 & 41,8 & 193,0 & 29,4 \\
\hline
\end{tabular}

$\mathrm{HP}$ - hospitais privados; $\mathrm{HO}$ - hospitais oficiais

Fonte: INE (2015) ${ }^{28}$

ligeiríssima subida na proporção destas unidades, ao passo que na região de Lisboa e Vale do Tejo este crescimento é mais acentuado. Em oposição, na região Centro verifica-se uma descida na percentagem total de hospitais públicos do país.

\section{Distribuição geográfica do número de camas disponí- vel para internamento nos hospitais}

Para reforçar a comparação público privada, após caraterizar a evolução do número de unidades públicas e privadas no sistema de saúde, analisa-se o comportamento da oferta pública/privada em termos da lotação (número de camas). De acordo com os dados disponíveis no INE, ${ }^{28}$ entre os anos 2004 e 2012, considerando as unidades de uma forma global atendendo apenas à propriedade, observa-se que os hospitais públicos reduziram a oferta de camas e que os hospitais privados investiram neste campo. A Tabela 3 reflete a distribuição do número de camas disponíveis nos hospitais portugueses distribuídas pela propriedade das unidades e pelas cinco regiões administrativas.

Com base nos dados apurados, apresentam-se algumas elações relativas à distribuição geográfica da lotação (por 100000 habitantes) dos hospitais públicos e privados:

- No setor privado, entre os anos 2004 e 2012 observou-se um crescimento global do número de camas no Algarve $(+58,3 \%)$, no Alentejo ( $+29,2 \%)$, na região Norte $(+25,1 \%)$ e em Lisboa $(+10,5 \%)$. A região centro foi a única em que se registou uma redução da aposta privada em $9,3 \%$.

- No setor público, entre os anos 2004 e 2012, registou-se uma redução do número de camas disponíveis para internamento em Lisboa $(-17,1 \%)$, na região Centro $(-10,8 \%)$, na região Norte $(-10,2 \%)$ e no Alentejo $(-4,5 \%)$. Apenas se registou um aumento da lotação na região do Algarve (+15,1\%).

\section{Considerações finais}

Nos últimos trinta anos desenvolveu-se uma relação crescente entre o setor público e o setor privado, enquadrada pela legislação aplicável ao setor da saúde, através da celebração de acordos, contratos de serviços, convenções e parcerias público-privadas. O aprofundamento desta combinação público-privada deveu-se em parte ao crescimento da oferta assegurada pelos prestadores privados nas últimas duas décadas.

Entre 2002 e 2012 os hospitais oficiais, de direito público, revelaram uma tendência de redução do número de unidades, em cerca de $7,6 \%$, por extinção ou por fusão de unidades de pequena e média dimensão, enquanto os hospitais privados aumentaram esse número em 10,6\%. Desta forma, no final de 2012 existiam, em Portugal, 110 hospitais oficiais e 104 hospitais privados. Igual tendência se assistiu quando analisada a evolução da lotação, em termos do número de camas, disponíveis em ambos os setores. As unidades públicas apresentam uma redução do número de camas e as privadas registam uma aposta neste campo. No entanto, destaca-se uma exceção na região centro, na qual ambos os setores reduziram a sua oferta.

Em termos da distribuição geográfica das unidades hospitalares, registou-se uma concentração dos prestadores privados nas regiões com maior densidade demográfica e rendimento per capita, em particular, na região Norte do país e em Lisboa e Vale do Tejo. No Algarve, região com um padrão demográfico variável de caráter sazonal, a oferta privada igualou a pública em termos do número de hospitais.

\section{CONFLITO DE INTERESSES}

Os autores declaram não ter nenhum conflito de interesses relativamente ao presente artigo.

\section{FONTES DE FINANCIAMENTO}

Não existiram fontes externas de financiamento para a realização deste artigo.

\section{REFERÊNCIAS}

1. Fernandes A. A combinação público-privado. In Campos A, Simões J, editors. 40 anos de abril na saúde. Coimbra: Almedina; 2014, p.213231.
2. Simões J, Campos A. O percurso da saúde: Portugal na Europa. Coimbra: Almedina; 2011.

3. Barros P. Transformar para servir melhor. In Fernandes J, Barros P, 


\section{8}

2009

2010

2011

2012

$\mathrm{HO}$

HP

$\mathrm{HO}$

HP

$\mathrm{HO}$

HP

$\mathrm{HO}$

HP

$\mathrm{HO}$

HP

$\begin{array}{rrr}207,1 & 75,0 & 206,2 \\ 297,4 & 58,1 & 289,6 \\ 287,8 & 116,5 & 286,3 \\ 185,7 & 30,4 & 180,8 \\ 181,8 & 23,1 & 184,7\end{array}$

80,1

211,1

81,9

207,3

86,8

207,3

289,6 55,2

284,2

52,7

280,6

116,4

276,8

45,6

280,8

$286,3 \quad 118,0$

283,3

118,1

273,0

$28,5 \quad 184,8$

40,2

183,6

$24,3 \quad 189,2$

33,7

219,9

Fernandes A, editors. Três olhares sobre o futuro da saúde em Portugal. Cascais: Princípia Editora; 2011. p. 51-82.

4. Oliveira M. Evolução da oferta. In Simões J, editor. 30 anos do Serviço Nacional de Saúde, um percurso comentado. Coimbra: Almedina; 2010, p.271-96.

5. Lei Constitucional $n \cdot{ }^{\circ} 1 / 2005$ - sétima revisão constitucional. Diário da República n. ${ }^{\circ}$ 155/2005 - I Série A, 12 de agosto de 2005, p. 4642.

6. Marques M, Moreira V. Desintervenção do Estado, privatização e regulação dos serviços públicos. Economia Perspectiva.1999;3:133-58.

7. Gouveia M. Política de Saúde. In Gouveia M, Pereira A, editors. Estratégias de Reforma do Estado Providência. Lisboa: Fórum de Administradores de Empresas; 1997. p. 21-156.

8. Saltman R. Patient choice and patient empowerment in Northern european health systems: a conceptual framework. Int J Health Serv. 1994;24:201-29.

9. Simões J. Retrato Político da Saúde - dependência do percurso e inovação em saúde: Da ideologia ao desempenho. Coimbra: Almedina; 2009.

10. Barata A, Tokura $\mathrm{Y}$, Martins $\mathrm{H}$. Comparing healthcare systems: considering Japan and Portugal. Rev Port Saúde Pública. 2012;30:18092.

11. James A, Stoskopf, C. Comparative health systems: global perspectives. Massachusetts: Jones and Bartlett Publishers. 2010.

12. Barros $P$, Machado M, Simões J. Portugal: health system review. Health systems in transition. OECD Publishing. 2011.

13. Inquérito nacional de saúde INS. 2005.

14. Dinis A. Saúde privada, integração sofisticada. Revista IP Espaços, edifícios e empresas. 2008:14-22.

15. Ribeiro J. Saúde, a liberdade de escolher. Lisboa: Gradiva; 2009.

16. Doyle Y, Bull A. Role of private sector in United Kingdom healthcare system. BMJ. 2000;321:563-65.

17. Tountas $Y$, Karnaki P, Pavi E, Souliotis K. The unexpected growth of the private setor in Greece. Health Policy. 2005;74:167-80.
18. Simões J. As parcerias público-privadas no setor de saúde em Portugal. Rev Port Saúde Pública. 2004;4:79-90.

19. Fernandes A. Mudar o presente para garantir o futuro. In Fernandes J, Barros P, Fernandes A, editors. Três olhares sobre o futuro da saúde em Portugal. Cascais: Princípia Editora; 2011. p. 83-98.

20. Beckfield J, Olafsdottir S, Sosnaud B. Healthcare systems in comparative perspective: classification, convergence, institutions, inequalities, and five missed turns. Ann Rev Sociol. 2013;39:127-46.

21. Instituto Nacional de Estatística. Estatísticas da saúde - inquérito aos Hospitais (2002-2012). Lisboa: INE. 2014.

22. Vaz A. Hospitais públicos portugueses. In Simões J, editor. 30 Anos de Serviço Nacional de Saúde: um percurso comentado. Coimbra: Almedina; 2010. p. 297-353.

23. PORDATA. Estatísticas dos Hospitais: números e camas. (2013) [consultado 2015 fev 15] disponível em http://www.pordata.pt/Portugal/ Hospitais.

24. Campos A. Reformas da saúde: o fio condutor. Coimbra: Almedina; 2008.

25. Ferreira D, Marques R. Did the corporization of portuguese hospitals significantly change their productivity? Eur J Health Econ. 2015;16:289303.

26. Rego G, Nunes R, Costa J. The challenge of corporatisation: the experience of portuguese-public hospitals. Eur $\mathrm{J}$ Health Econ. 2010;4:367-81.

27. Azevedo $\mathrm{H}$, Mateus, C. Economias de escala e de diversificacão: uma análise da bibliografia no contexto das fusões hospitalares. Rev Port Saúde Pública. 2014;32:106-17.

28. Instituto Nacional de Estatística. Estatísticas da saúde - Camas dos hospitais por 100000 habitantes $\left(\mathrm{N} .^{\circ}\right.$ ) por Localização geográfica (NUTS - 2013), Natureza institucional. [consultado 2015 set 20] Disponível em: https://www.ine.pt/xportal/xmain?xpid=INE\&xpgid=ine_ indicadores\&indOcorr Cod=0008100\&contexto=bd\&selTab=tab2. 